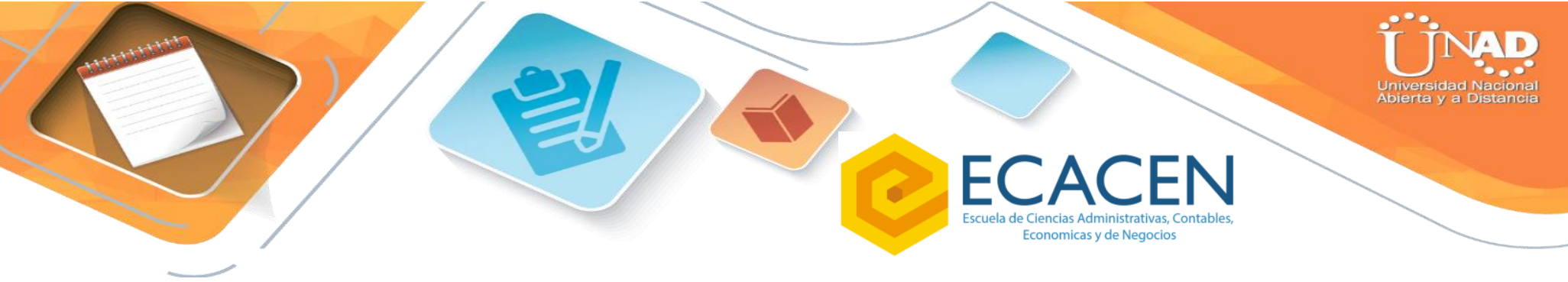

ESTADO DEL ARTE DEL TELETRABAJO

\title{
STATE OF THE ART OF TELEWORKING
}

\author{
Nancy Amparo Guaca ${ }^{1}$ \\ Yudith Caicedo ${ }^{2}$ \\ Juan Carlos Díaz Dávila ${ }^{3}$
}

\section{Resumen}

En el presente documento se realiza inicialmente un recorrido por el concepto de teletrabajo desde diferentes autores, seguido de exponer mediante cifras, estudios realizados, la tendencia tanto en Europa como en Estados Unidos frente a esta modalidad de trabajo que cada vez coge más fuerza y genera mayor interés entre los empresarios y el estamento gubernamental debido entre otros factores al acceso creciente a las tecnologías de la información y la comunicación Tics. De igual manera se aborda la revisión de cifras del teletrabajo en América Latina y en especial para Colombia, enfatizando en estudios realizados en el país frente a la viabilidad e implementación, así como experiencias de éxito en el entorno colombiano y finalmente se aborda la posición, interés y avance gubernamental frente al tema.

\footnotetext{
${ }^{1}$ Magister en Administración de Organizaciones, Especialista en Redes y Comunicaciones, Candidata a Magister en Telecomunicaciones, Docente de la Universidad Nacional Abierta y a Distancia UNAD, nancy.guaca@unad.edu.co.

2 Magister en Administración de Organizaciones, Especialista en Gestión del Talento Humano, Docente Universidad Nacional Abierta y a Distancia UNAD, Yudith.caicedo@unad.edu.co.

3 Administrador de Empresas, Especialista en Gerencia de Negocios Internacionales Magister en Administración de Empresas. Docente de la Escuela de Ciencias Administrativas Contables Económicas y de Negocios ECACEN de la UNAD, investigador grupo Sindamanoy, líneas de investigación: emprendimiento y cadenas productivas. Correo electrónico juanc.diaz@unad.edu.co, C.C. No. 12’992.912. Código ORCID https://orcid.org/0000-0001-7824-6113
} 


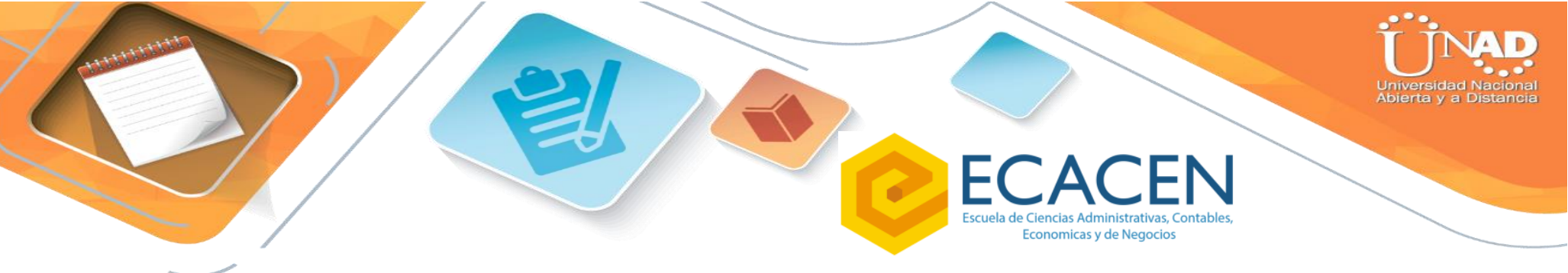

familia, lactantes o gestantes, residentes en zonas rurales apartadas. El Gobierno Nacional ha planteado un modelo para su implementación consignado en el Libro Blanco. El ABC del teletrabajo en Colombia.

\section{Estado del Arte}

En el artículo Estrategias de mejora de la salud psicosocial del teletrabajador el arte de conjugar teoría y práctica, señala que el origen del término aparece en los Estados Unidos con Jack M. Nilles, lo define como la actividad de trabajar a distancia como "telecommuning", este término en castellano se puede traducir como "teledesplazamiento", se hace referencia a que es el trabajo el que se desplaza hasta el trabajador y no viceversa. Desde entonces, el término "telecommuting" se ha empleado en Estados Unidos, el término usado en Europa es "telework" teletrabajo. (Gallego, 2008)

Mercedes, (2013) en su ponencia: La Sociedad de la Información señala que "la ruptura de la fórmula empleado-oficina, desplazándose el trabajo hacia el teletrabajador, se inicia en los años 70, con la crisis del petróleo en los Estados Unidos" evitando el consumo de combustible y los desplazamientos a la oficina, razón por la cual se usa el término telecommuting o teledesplazamiento en vez de teletrabajo.

Como lo indica Gallego (2008) en el artículo una definición clásica del teletrabajo como un modo de empleo en el que el trabajador está localizado remotamente de una oficina central o de un centro de producción, con o sin contacto cara a cara con co-trabajadores, pero que permite la comunicación vía el uso de la tecnología de sistemas de comunicación (Conner, Fletcher, Firth-Conzens y Colling, 1994). Padilla en 1997 indica que teletrabajo es una estrategia funcional de una organización, basada en trabajar cuando quiera y en cualquier parte, haciendo los Recursos Humanos, por tanto, más flexibles.

Arias Hidalgo (2015) señala en el marco de la Jornada de Telework Week 2015 que en el mundo se estima que hay 1.300 millones de teletrabajadores. 
En el artículo el teletrabajo en Aragón: estudio de su adopción potencial en las empresas, que para el año 1999 había en Europa unos nueve millones de teletrabajadores, se observa un crecimiento importante desde 1997, donde se registraban solamente dos millones de teletrabajadores. Para el año 1999 en Estados Unidos contaba con 15, 7 millones y en Japón 2.1 millones de teletrabajadores. Resalta el artículo que en la Unión Europea el mayor número de teletrabajadores se encuentran en Alemania, Reino Unido y Holanda. El artículo relaciona además la relación a la población ocupada el porcentaje de teletrabajadores respecto a la fuerza laboral es mayor en los países Nórdicos: Finlandia $(16,7 \%)$, Suecia $(15,1 \%)$, Holanda $(14,5 \%)$, Dinamarca $(10,4 \%)$, España $(2,8 \%)$ porcentaje por debajo de la media europea que es del 6\%. (Pérez, Luis Carnicer, \& Martinez Sánchez, 2001).

En el documento titulado Overview on European policies on TELEWORK presenta una tabla que resume el desarrollo del teletrabajo en algunos países de la Unión Europea en los años 1999 y 2005. (Baltina, 2015)

Tabla 1. Teletrabajo en Unión Europea

\begin{tabular}{|l|c|c|c|}
\hline \multicolumn{1}{|c|}{ País } & $\mathbf{1 9 9 9}$ & \multicolumn{2}{c|}{2005} \\
\hline Teletrabajadores & $\begin{array}{c}\text { Teletrabajadores al } \\
\text { menos un cuarto de } \\
\text { tiempo o más }\end{array}$ & $\begin{array}{c}\text { Teletrabajadores } \\
\text { casi todo el tiempo }\end{array}$ \\
\hline Dinamarca & 10.48 & 14.4 & 2.6 \\
\hline Países Bajos & 14.53 & 12 & 1.9 \\
\hline Reino Unido & 7.62 & 8.1 & 2.5 \\
\hline España & 2.81 & 6.9 & 1.5 \\
\hline Francia & 2.87 & 5.7 & 0.5 \\
\hline Italia & 3.59 & 2.3 & 1.6 \\
\hline
\end{tabular}

Fuente: Overview on European policies on TELEWORK 


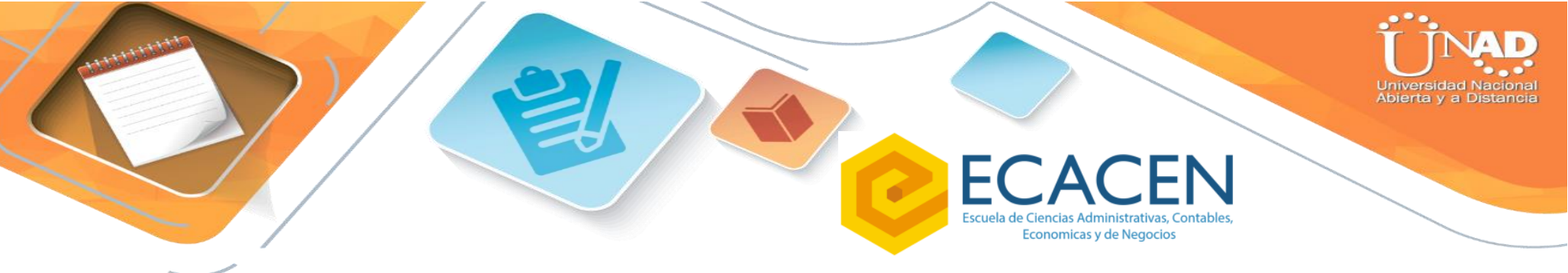

En Europa, durante los años '90, hubo un crecimiento de experiencias de teletrabajo en varios países. En el mes de julio de 2002, los actores sociales europeos (Confederación Europea de Sindicatos -CES, Confederación de Empresas Europeas UNICE, Centro Europeo de Estrategia Política - CEEP) negociaron el Acuerdo Marco Europeo de Teletrabajo que se convirtió en el principal punto de referencia para todas las experiencias sucesivas a nivel nacional. La idea de fondo es posibilitar el mismo nivel de protección tanto a los trabajadores presenciales como a los teletrabajadores. (UNED, 2002)

Worldatwork es una asociación profesional sin ánimo de lucro fundada en 1955, esta entidad expidió Telework 2011. En el documento WorldatWork Special Report, presenta una revisión del teletrabajo en los Estados Unidos, esta organización ha realizado el estudio del teletrabajo desde el año 2003 y por primera vez se observa que el número de teletrabajadores ha disminuido: el informe indica que el número de teletrabajadores por un día o al menos una vez al mes fue en el año 2010 de 26,2 millones, frente a los 33,7 millones en 2008. La cifra de 26 millones, representa cerca del $20 \%$ de la población adulta que trabaja en los Estados Unidos de 139 millones (a partir del cuarto trimestre de 2010 (BLS 2011)). A primera vista, se puede llegar a concluir que el teletrabajo se estancó, sin embargo, la disminución indica el organismo puede ser probablemente por la alta tasa de desempleo, falta de conocimiento de las opciones de teletrabajo entre otros factores. (Worldatwork, 2011).

En la siguiente figura se observa la tendencia del teletrabajo las cifras están en millones.

Figura 1. Tendencia del teletrabajo Estados Unidos 


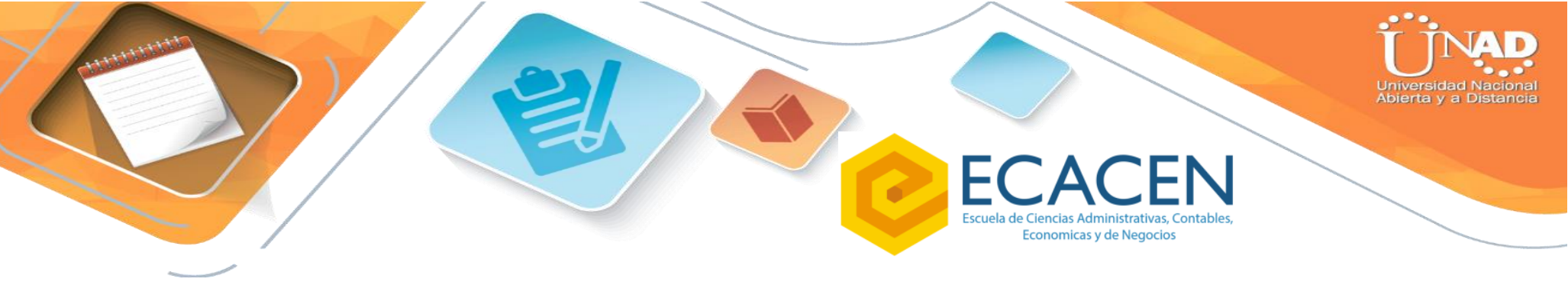

Brasil mediante el proyecto de ley 5405 de 2008 "Se reglamenta el teletrabajo a distancia, conceptúa y disciplina las relaciones de teletrabajadores".

Herrera revisa las cifras que plasma HowDoIBecomeA.net una plataforma que examina el mundo del trabajo desde un ángulo global, de una estimación al escenario latinoamericano. Los números indican que como región (tomando en cuenta México, Brasil y Argentina) el promedio de personas que teletrabaja al menos una vez a la semana supera a países europeos e incluso, a los Estados Unidos: (Herrera , 2013)

> México: el 58\% de los trabajadores teletrabaja al menos una vez a la semana, mientras un $6 \%$ es un teletrabajador full time.

$>$ Brasil: $28 \%$ de los trabajadores teletrabaja al menos una vez a la semana, mientras un $9 \%$ es un teletrabajador full time.

> Argentina: un 45\% de los trabajadores teletrabaja al menos una vez a la semana, mientras un $9 \%$ es un teletrabajador full time.

En el artículo, Herrera menciona las estadísticas de Freelancer.com, que es una bolsa online en el que se puede solicitar servicios de diferentes ramas y destinado a la pequeña y mediana empresa, señala que Argentina junto con Brasil son los países con mayor cantidad de usuarios, le siguen México, Colombia, Chile y Perú. Los profesionales argentinos que se registran son principalmente en el área relacionada con la tecnología, manejo de más de un idioma, y la alta conectividad existente, hacen de los argentinos candidatos altamente buscados por los empleadores. Revisa el informe además el uso promedio de internet donde Argentina lidera en la región con un promedio mensual de 27,4 horas por visitante, luego se ubica Brasil con 25,4 horas y México con 25,1 horas, también superando el promedio mundial de 23,1 horas al mes por visitante. (Herrera, 2013).

\section{Teletrabajo en Colombia}

Arias Hidalgo (2015) en el marco de la Jornada de Telework Week 2015 señala que en el Gobierno Colombiano ha firmado 205 pactos con empresas públicas y privadas, se cuenta con 47.000 teletrabajadores y se proyecta para el 2018 una meta 


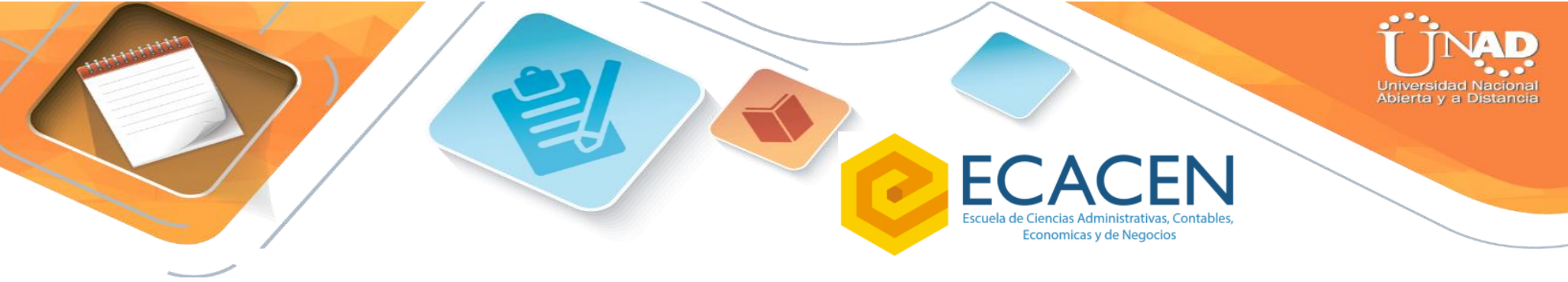

de 120.000. Entre esos pactos en portal de Colombia digital se observan experiencias exitosas en organizaciones como Edatel, Contraloría de Antioquia, entre otras.

El portal de teletrabajo Colombia realiza una reseña del primer foro Telework Week, realizado el 16 de septiembre 2015 en la ciudad de Medellín, en el marco del Día Internacional del Teletrabajo: se contó con la asistencia de 500 personas, conferencistas internacionales, representantes del gobierno colombiano, la empresa privada y miembros de la academia. Se reveló los resultados de la primera Semana de Teletrabajo realizada entre el 24 al 28 de agosto del 2015: se destacó que cada empleado que adoptó la modalidad de teletrabajo ahorra cerca de 6.5 horas en desplazamiento (equivalente a una jornada diaria labora), de los 48 mil teletrabajadores durante la semana ahorrarían cerca de 14 millones de galones de combustible. (Portal Teletrabajo Colombia, 2015)

El portal de teletrabajo de Colombia, señala que en el primer foro Telework Week, quedó ampliamente respaldada por el estudio MinTic y Nubelo (plataforma de servicios Freelance líder en países de habla hispana). El argentino Jorge Araujo, director ejecutivo de la compañía, reveló en su ponencia que siete de cada diez trabajadores digitales han aumentado sus ingresos un $66 \%$, y seis de cada diez personas están satisfechos o muy satisfechos con esta modalidad de trabajo. (Portal Teletrabajo Colombia, 2015)

En Colombia varias compañías firmaron el pacto del teletrabajo, impulsando esta modalidad laboral en sus organizaciones, se suman a las 205 empresas que han pactado con MINTIC el apoyo a esta iniciativa, entre las organizaciones firmantes se encuentran: AIG Services Latin America S.L, el Banco Agrario de Colombia, la Universidad de Medellín, la Cámara de Comercio de Sincelejo, entre otras. Los conferencistas internacionales invitados, ratifican el buen camino del teletrabajo en Colombia, pero también el gran reto que tienen de seguir avanzando, al ser un punto de referencia en el tema para toda la región. (Portal Teletrabajo Colombia, 2015)

Entre las experiencias exitosas relacionadas en el portal Colombia Digital Adriana Molano relacionan el caso de Edatel (empresa de soluciones integrales de tecnologías de información y las comunicaciones), con más de cuarenta años en el 
Así, Alastruey (2013), en el documento: El networking y las redes sociales, la perfecta herramienta para el teletrabajo, formula propuestas para el teletrabajador a fin de mejorar la calidad y rentabilidad de los puestos de trabajo.

La autora resalta que se requiere profesionales proactivos, que tengan iniciativa, constancia, esfuerzo, responsabilidad y actitud emprendedora, siendo estas algunas competencias-habilidades profesionales que empiezan a solicitar en el nuevo mercado laboral. Uso de herramientas de comunicación colectiva (como Messenger, Skype, videoconferencia, entre otros) y/o repositorios y compartición de conocimiento (YouTube, Prezi, SlideShare, entre otros) que permitan estar interactuando entre clientes, equipos y profesionales que caracteriza y pide el teletrabajo.

García Palomino en el documento Guía de aplicación de la Norma OHSAS 18001: 2007 para el teletrabajo en las organizaciones, revisa el sistema de gestión de la Seguridad y Salud ocupacional en el teletrabajo, basado en el estándar Occupational Health and Safety Assessment Series - OHSAS. Realiza una guía de aplicación teniendo en cuenta la normatividad a fin de garantizar condiciones de seguridad y salud ocupacional en el teletrabajo se realice teniendo en cuenta normas internacionales. (Garcia Palomino, 2013),

En su tesis de grado para optar al título como Magister en Desarrollo Humano Organizacional, titulada: Riesgos Sociales en el Teletrabajo, Moreno y Jiménez analizan en su estudio el factor de riesgo psicosocial debido al estrés, teniendo en cuenta la resolución 2646 del 2008, revisa las empresas antioqueñas pioneras en la implementación del teletrabajo y sus implicaciones en el teletrabajador. En este análisis destacan unidades de análisis entre las que se encuentran: aislamiento físico, exigencias cognitivas (incorporación de nuevas tecnologías), equilibrio vida personal vs vida laboral, repercusiones en la salud. De las cinco empresas involucradas en la investigación, cuatro de ellas implementan el teletrabajo por intereses en términos de estructura y racionalización de costos. Indica además que cuando la empresa no cuenta con una plataforma tecnológica adecuada, genera ansiedad, tensión y exceso de trabajo represado, que conlleva impacto negativo del teletrabajador. (Moreno De Luca \& Jiménez Prada, 2013) 
El estudio concluye que las administradoras de Riesgos Laborales (ARL) delegan la responsabilidad sobre las empresas quienes son las encargadas de evaluar, medir y actuar sobre los riesgos laborales, por su parte los teletrabajadores son responsables de su autocuidado y les corresponde cumplir las acciones recomendadas por las ARL. El estudio detecta efectos en el proceso de socialización, sensación de aislamiento, esfuerzo cognitivo por el uso de herramientas tecnológicas, exigencia de desarrollar nuevas competencias en manejo de las Tics, entre otros aspectos.

Delgado y Jimenez, docentes de la Universidad Externado en su investigación percepciones sobre el Teletrabajo en Colombia ponen a consideración y analisis lo siguiente: (Delgado Wiesner \& Jiménez Villamizar, 2014)

"El Teletrabajo en Colombia es una modalidad laboral reciente y aún desconocida para gran parte de los integrantes del proceso productivo del país. Por esta razón se consideró necesario realizar una investigación exploratoria sobre el conocimiento y las percepciones que se tienen sobre el mismo y sus posibilidades de implementación. El trabajo se enfoca desde una perspectiva socio-cultural, y se refiere exclusivamente a la percepción sobre el teletrabajo corporativo, cuando éste se realiza desde el domicilio".

Los hallazgos muestran una percepción positiva hacia este tipo de trabajo, aunque no se considera la factibilidad de realizar todas las funciones mediante esta modalidad, el factor de interacción personal y problemas de sociabilidad igualmente inciden en las preocupaciones expuestas por el grupo encuestado. La concepción funcional del teletrabajo se asocia más con valores que con destrezas tecnológicas y organizativas, y la movilidad no es considerada como factor determinante para la adopción del teletrabajo"

La investigaciòn resalta que para teletrabajador se requiere ademàs del conocimiento profesional, un perfil que reúna una serie de cualidades y valores como la disciplina, responsabilidad y autonomía; la investigación indica que las destrezas tecnológicas se asumen como inherentes y no constituye un requisito para teletrabajar. En la población encuestada existe una percepciòn positiva de la dinámica del teletrabajo, la mayoría estàn dispuestos a cambiar su modalidad de trabajo. 


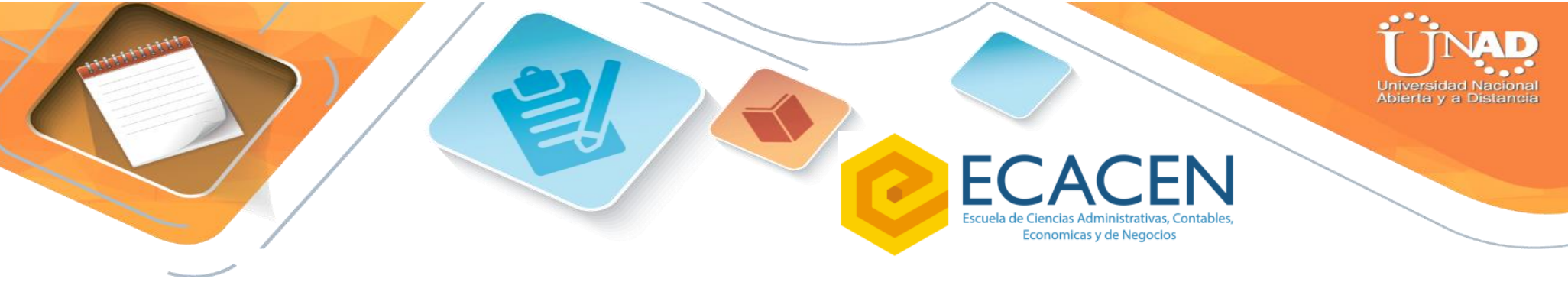

Baltina, I. (2015). Overview on European policies on TELEWORK. Latvia: Riga Technical University.

Beltran Puche, A., \& Sanchez Neira, L. (2002). Las relaciones laborales virtuales:

Teletrabajo. (P. U. Javeriana, Recopilador) Bogotá. Obtenido de Las relaciones laborales virtuales: Teletrabajo:

http://javeriana.edu.co/biblos/tesis/derecho/dere4/Tesis-31.pdf

Buitrago Rodríguez, J. (2015). Universidad Militar Nueva Granada . Obtenido de Propuesta del plan piloto para la implementación del teletrabajo en el centro militar penitenciario de Puente Aranda como opción de trabajo y redención de pena.: Obtenido de:

http://repository.unimilitar.edu.co:8080/bitstream/10654/13947/2/Monografia \%20Teletrabajo\%20Jhonny\%20Buitrago\%2018-03-15.pdf

Camacho Peláez , R., \& Higuita Lopez , D. (2013). Teletrabajo con calidad de vida laboral y productividad. Una aproximación a un modelo en una empresa del sector energético. Pensamiento y Gestión, 35, 87-118. Obtenido de: http://www.scielo.org.co/pdf/pege/n35/n35a05.pdf

Colombia Digital . (Septiembre de 2013). Teletrabajo eLAC 2015: ¿Cómo va Argentina? Obtenido de: http://colombiadigital.net/teletrabajo/item/5711-teletrabajo-elac2015-como-va-argentina.html

Chirinos Aita, E., \& Panta Escurra , A. (Julio de 2014). Universidad Católica Santo Toribio de Mogrovejo. Obtenido de Potencial del teletrabajo en la Universidad Católica Santo Toribio de Mogrovejo.: Obtenido de: http://54.165.197.99/jspui/bitstream/123456789/303/1/TL_ChirinosAitaElsa_P antaEscurraAngela.pdf

Delgado Wiesner, C., \& Jiménez Villamizar, J. (2014). Percepciones sobre el teletrabajo en Colombia. Obtenido de Telework2010.

Gallego, E. (2008). Estrategias de mejora de la salud psicosocial del teletrabajador el arte de conjugar teoría y práctica. Revista de trabajo y seguridad social, 181200. 


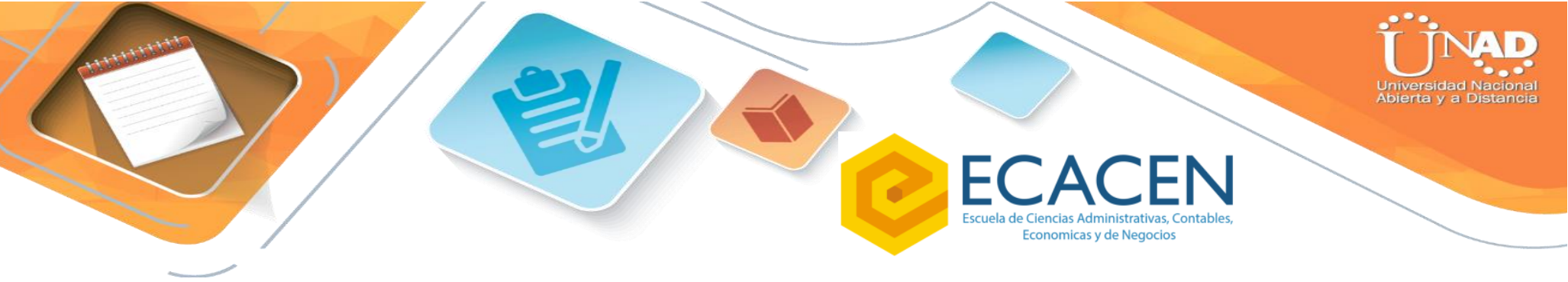

Garcia Palomino, M. (2013). Guía de aplicación de la Norma OHSAS 18001: 2007 para el teletrabajo en las organizaciones. Obtenido de Universidad Militar Nueva Granada: Obtenido de:

http://repository.unimilitar.edu.co:8080/bitstream/10654/10419/1/GarciaPalo minoMaribel2013.pdf

Herrera , C. (21 de Junio de 2013). En América Latina, el promedio de teletrabajo supera a Europa y Estados Unidos. Obtenido de http://pulsosocial.com/2013/06/21/en-america-latina-el-promedio-deteletrabajo-supera-a-europa-y-estados-unidos/

Mercedes, P. (2013). Universidade Federal Fluminense. Obtenido de Universidad Carlos III de Madrid: Obtenido de: http://repositorios.questoesemrede.uff.br/repositorios/bitstream/handle/12345 6789/932/La\%20sociedad\%20-\%20Mercedes.pdf?sequence=1

MINTIC. (22 de Octubre de 2013). Ser un teletrabajador competente. Obtenido de http://www.mintic.gov.co/portal/604/w3-article-4553.html

Molano, A. (2012). Colombia Digital . Obtenido de Edatel y el teletrabajo: Obtenido de: http://colombiadigital.net/herramientas/item/1778-edatel-y-el-teletrabajo.html

Moreno De Luca, N., \& Jiménez Prada, C. (2013). Universidad EAFIT. Obtenido de Riesgos psicosociales en el teletrabajo: Obtenido de: https://repository.eafit.edu.co/bitstream/handle/10784/2669/MorenoDeLuca_N athalia_2013.pdf?sequence $=1$ \&isAllowed $=y$

Pérez, M., Luis Carnicer, M., \& Martinez Sánchez, Á. (2001). El teletrabajo en Aragón: estudio de su adopción potencial en las empresas. Publicación trimestral de la Caja de Ahorros y MP de Zaragoza, Aragón y Rioja., 107-132.

Portal Teletrabajo Colombia. (2015). Especial del Foro Telework Week. Obtenido de: http://teletrabajo.gov.co/portal/13747:

Querol , V., \& Sáez, E. (2013). Ideas, tendencias y nuevas formas de trabajo para la economía del conocimiento. Obtenido de: http://www3.uji.es/ soro/Teletrabajo.pdf 
Ríos Hurtado, M., Flórez Pineda, J., \& Rodríguez, L. (2012). Universidad EAN. Obtenido de Biblioteca Digital Minerva:

http://repository.ean.edu.co/bitstream/handle/10882/2963/FlorezJose2012.pdf ?sequence $=1$ \&isAllowed $=y$

Sánchez Galvis , M. (2012). Comisión Económica para América Latina y el Caribe Cepal . Obtenido de Un acercamiento a la medición del teletrabajo: Evidencia de algunos países de América Latina: Obtenido de:

http://repositorio.cepal.org/bitstream/handle/11362/3966/S1200081_es.pdf?se quence $=1$

UNED, U. E. (2002). Obtenido de Acuerdo Marco Europeo sobre Teletrabajo: http://www.uned.ac.cr/viplan/images/acuerdo-marco-europeo-sobreteletrabajo.pdf

Worldatwork. (2011). Telework 2011. A WorldatWork Special Report. Obtenido de http://www.worldatwork.org/waw/adimLink?id=53034 